\title{
ETUDE DE L'EFFICACITÉ RELATIVE DES DIVERSES FORMULES DE BARYMÉTRIE APPLICABLES AUX BOVINS
}

PAR

\section{J. DELAGE, J. POLY, B. VISSAC}

(Laboratoire de Recherches de Zootechnie, Institut National Agronomique, Paris).

\section{PLAN DU MEMOIRE}

\section{I. - Introduction; exposé du problème.}

II. - Revue des travaux antérieurs :

I $^{\mathrm{O}}$ Relation entre le poids vif et le tour de poitrine.

$2^{\circ}$ Causes de variation du coefficient de régression du poids vif sur le tour de poitrine.

$3^{o}$ Équations de régression du poids vif sur le tour de poitrine.

$4^{\text {o }}$ Estimation pratique du poids vif d'après le tour de poitrine.

$5^{\circ}$ Sources d'erreurs résultant de l'emploi des formules de barymétrie.

$6^{\circ}$ Autres relations proposées par divers auteurs.

\section{III. - Résultats obtenus à l'aide de nos données :}

Io Corrélation entre le poids vif et certaines mensurations corporelles.

$2^{\circ}$ Coefficients de régression linéaire du poids vif sur le tour de poitrine, le tour spiral et leur moyenne arithmétique.

$3^{\circ}$ Valeur statistique des trois équations de régression linéaire.

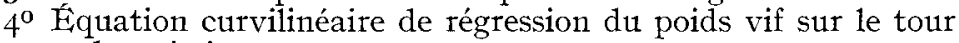
de poitrine.

$5^{\circ}$ Comparaison des régressions linéaire et curvilinéaire du poids vif sur le tour de poitrine.

\section{IV. - Conclusions.}

V. - Bibliographie.

\section{I. - INTRODUCTION}

La zoométrie, chez les bovins, a souvent revêtu, dans le passé, un aspect purement théorique ; cependant, depuis quelque temps, les mensurations tendent à connaître en élevage, un regain d'intérêt, en raison de leurs applications pratiques très variées. C'est ainsi que les Livres Généalogiques cherchent à en généraliser l'enregistrement pour leurs 
animaux inscrits. I.es nombreux travaux qui leur ont été consacrés peuvent se classer sous 3 rubriques différentes:

Mensurations et Poids vif. - Beaucoup d'études ont eu pour but d'établir des relations mathématiques entre certaines mensurations et le poids vif des animaux, et plusieurs formules de barymétrie ont été proposées.

Mensurations et Conformation chez l'adulte. - I,es auteurs ont défini, soit à l'échelon de l'individu, les caractères de l'animal par des mesures chiffrées, soit à l'échelon de la race, un standard de conformation ou à 1'échelon de la population, un type d'animal.

Mensurations et Croissance. - Il parait logique que de nombreuses recherches sur les mensurations aient été effectuées au cours de la croissance du jeune ; c'est, en effet, durant cette période cruciale de la vie de l'animal que se produisent d'importantes modifications morphologiques de son organisme, dont la résultante la plus évidente est la variation de son poids vif.

Nous nous limiterons, dans cette première communication, à l'étude des problèmes de barymétrie.

\section{Mensurations et Barymétrie}

La détermination rapide et précise du poids vif des bovins a sourent posé à la majorité des éleveurs le problème de l'achat d'une bascule. Cette connaissance du poids vif devient pourtant, de plus en plus nécessaire devant les impératifs des techniques modernes de l'élevage :

a) Détermination des besoins d'entretien des animaux et contrôle de l'efficacité du rationnement.

b) Estimation des variations de poids au cours d'une lactation ou d'un exercice comptable.

c) Contrôle de l'aptitude à la production de la viande.

d) Appréciation des poids vifs sur les lieux des Concours.

Ces observations justifient les nombreuses recherches consacrées à l'étude des relations existant entre le poids vif et certaines mensurations corporelles.

\section{II. - LES TRAVAUX ANTÉRIEURS}

Après avoir déterminé les corrélations existant entre le poids vif et certaines mensurations corporelles fondamentales (hauteur au garrot, longueur du corps, tour de poitrine, etc....) ou des combinaisons de ces mensurations, les auteurs ont conclu que c'est, en général, le tour de poitrine qui présentait le plus gros intérêt. 


\section{$1^{0}$ Les relations entre le poids vif et le tour de poitrine :}

Le tableau I donne un aperçu des corrélations obtenues par différents auteurs et rapportées par JoHANSSON et HILDEMAN (I). Les coefficients de corrélation cités sont très voisins et supérieurs à 0,9 .

\section{TABLEAU I}

Corrélation entre le poids vif en $\mathrm{kg}$ et le tour de poitrine en $\mathrm{cm}$ (d'après JoHANsSon et Hildeman)

\begin{tabular}{|c|c|c|c|c|}
\hline Matériel d'expérience & $\begin{array}{l}\text { Nombre } \\
\text { d'animaux }\end{array}$ & $\begin{array}{l}\text { Coefficient } \\
\text { de } \\
\text { corrélation }\end{array}$ & $\begin{array}{c}\text { Coefficient } \\
\text { de régression } \\
\text { du poids vif } \\
\text { sur le tour } \\
\text { de poitrine }\end{array}$ & Référence \\
\hline $\begin{array}{l}\text { Génisses, vaches frisonnes Suédoises. } \\
\text { Taureaux, vaches, génisses de races }\end{array}$ & 712 & $0,95 \mathrm{I}$ & & Hansson (1926) \\
\hline 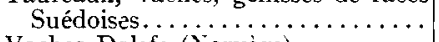 & 474 & 0,956 & $7,5^{8}$ & " $"$, \\
\hline Vaches Dolefe (Xorvège)........... & 973 & & 5,53 & Aasmundstad (I935) \\
\hline Génisses Dolefe (Norvège)......... & $\mathrm{I}_{4} \mathrm{I}$ & & 5,06 & $» \quad$ \\
\hline Taureaux Dolefe (Norvège)......... & 477 & & 7,50 & Clo $" 2$ \\
\hline Taureaux Red-Polled (Norvège).... & 282 & 0,92 & 8,83 & Slagsvold (I949) \\
\hline
\end{tabular}

Branton et Salisbury (2) travaillant sur 2 lots de 25 taureaux Guernesey et 25 taureaux Holstein ont trouvé entre le poids vif et le tour de poitrine des coefficients de corrélation de 0,958 et 0,954 respectivement.

\section{$2^{\circ}$ Les causes de variation du coefficient de regression du poids vif sur le tour de poitrine.}

Alors que les coefficients de corrélation sont très élevés et assez voisins, 'es coefficients de régression du poids vif sur le tour de poitrine varient par contre beaucoup plus et leurs variations sont affectées par le sexe, l'âge et l'état des animaux.

\section{a) L'infuence du sexe :}

Johansson et HiLdeman (I) constatent, par exemple, des différences importantes entre les sexes, comme le prouvent les données du tableau II, concernant des jeunes génisses et des jeunes taureaux de même âge. Il faut signaler que les coefficients de régression trouvés pour les mâles sont toujours supérieurs à ceux des femelles, c'est-à-dire que, pour une même augmentation du tour de poitrine, l'accroissement de poids est supérieur chez les mâles, ce qui peut s'expliquer par un meilleur développement, dans ce sexe, des masses musculaires et du squelette.

\section{b) L'influance de l'âge :}

Les résultats des auteurs précédents se rapportant aux jeunes veaux, jeunes génisses et génisses plus âgées indiquent, indiscutablement une 
augmentation, avec l'âge des animaux, du coefficient de régression du poids vif en $\mathrm{kg}$, sur le tour de poitrine en $\mathrm{cm}$.

Cela signifie qu'une même augmentation du tour de poitrine chez le jeune entraîne des accroissements de poids d'autant plus élevés que les animaux sont plus âgés. Ce fait est lié à l'allure curvilinéaire de la courbe de croissance.

\section{TABLEAU II}

Coefficient de régression du poids vif en $\mathrm{kg}$, sur le tour de poitrine en $\mathrm{cm}$ en fonction de l'âge et du sexe (d'après JoHANsson et HILdEMAN (I))

\begin{tabular}{|c|c|c|c|c|}
\hline & \multicolumn{2}{|c|}{ Race Pie rouge Suédoise } & \multicolumn{2}{|c|}{ Race Frisonne Suédoise } \\
\hline & $\begin{array}{l}\text { Nombre } \\
\text { de sujets }\end{array}$ & $\begin{array}{l}\text { Coefflcient } \\
\text { de régression }\end{array}$ & $\begin{array}{l}\text { Nombre } \\
\text { de sujets }\end{array}$ & $\begin{array}{l}\text { Coefflcient } \\
\text { de régression }\end{array}$ \\
\hline $\begin{array}{l}\text { Jeunes veaux } \ldots \ldots \ldots \ldots \ldots \ldots \\
\text { Jeunes génisses } \ldots \ldots \ldots \ldots \ldots \\
\text { Jeunes taureaux } \ldots \ldots \ldots \ldots \ldots \\
\text { Génisses plus âgées } \ldots \ldots \ldots \ldots \ldots\end{array}$ & $\begin{array}{l}780 \\
108 \\
107 \\
168\end{array}$ & $\begin{array}{l}0,82 \\
3,23 \\
6,03 \\
4,07\end{array}$ & $\begin{array}{r}152 \\
\text { I } 77 \\
64 \\
\text { I } 45\end{array}$ & $\begin{array}{l}0,99 \\
3,23 \\
6,28 \\
4,67\end{array}$ \\
\hline
\end{tabular}

c) L'infuence de l'état de l'animal, qui varie notamment avec :

L'état de gestation : L'état de gestation modifie évidemment les relations trouvées entre le poids vif et le tour de poitrine : les résultats d'OSTERGAARD (3) sont particulièrement suggestifs à cet égard. Cet auteur obtient des valeurs de 6,7 pour des Jerseyaises en gestation et de 5,6 pour des femelles, de la même race, non pleines.

Le niveau de l'alimentation: Le tableau III rassemble les résultats d'expériences réalisées par JoHANsson et HILDEMAN (I) sur 3 groupes de génisses soumises à 3 régimes alimentaires de niveaux différents : haut, moyen et bas.

\section{TABLEAU III}

Coefficient de régression du poids vif sur le tour de poitrine en fonction du niveau d'alimentation

(d'après Johansson et HII,DEMAN (I)).

\begin{tabular}{|c|c|c|}
\hline Niveau alimentaire & Génisses Pie rouge Sućdoises & Génisses Frisonnes Suédoises \\
\hline 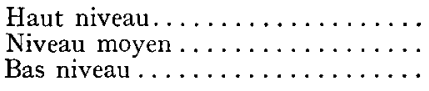 & $\begin{array}{l}5,29 \\
4,83 \\
4,63\end{array}$ & $\begin{array}{l}4,94 \\
4.67 \\
4,24\end{array}$ \\
\hline
\end{tabular}

Il est logique de constater un coefficient de régression plus élevé pour les génisses soumises à un haut niveau de nutrition, car le dévelop- 
pement des parties postérieures d'un bovin est considérablement modifié par son état d'engraissement.

STEENSBERG et OSTERGAARD (4) ont, par ailleurs, établi un tableau à double entrée avec lequel il est possible d'estimer le poids vif à partir du tour de poitrine, en tenant compte de 2 éléments correctifs : 1'âge exprimé en mois et le niveau alimentaire qui conditionne évidemment l'état d'embonpoint.

\section{$3^{\circ}$ Equation de Régression du poids vif sur le tour de poitrine.}

De nombreux auteurs, parmi lesquels BRODY, DAVIs et RAGSDALE (5) ont fait figure de pionniers, se sont attachés à définir la meilleure relation de la forme $\mathrm{P}=a \mathrm{~T}^{n}, \mathrm{P}$ étant le poids vif, $\mathrm{T}$ le tour de poitrine, $a$ et $n$ des constantes, donnant en fonction du tour de poitrine le poids de l'animal. Les principaux résultats enregistrés dans la bibliographie et rassemblés par Johansson et HildEman sont consignés dans le tableau IV. On constate que $n$ est un nombre voisin de 3 , ce qui a évidemment une signification biologique puisque le poids est le cube d'une dimension linéaire multiplié par une densité.

\section{TABLEAU IV}

Relations entre le poids vif en kg et le tour de poitrine en $\mathrm{cm}$ (d'après Johansson et HILDEMan (I)).

\begin{tabular}{|c|c|c|c|}
\hline Matériel d'expérience & $\begin{array}{c}\text { Nombre } \\
\text { d'animaux }\end{array}$ & Relation & Référence \\
\hline $\begin{array}{l}\text { Holstein } \\
\text { Jersey } \\
\text { Guernesey } \\
\text { Ayrshire } \\
\text { Vaches Red-Polled.......... }\end{array}$ & $\begin{array}{r}8320 \\
4993 \\
840 \\
1447 \\
\times 682\end{array}$ & $\begin{array}{l}\mathrm{P}=0,00029 \mathrm{~T}^{2,75} \\
\mathrm{P}=0,00019 \mathrm{~T}^{2,83} \\
\mathrm{P}=0,000 \mathrm{I} 3 \mathrm{~T}^{2,91} \\
\mathrm{P}=0,00014 \mathrm{~T}^{2,88} \\
\mathrm{P}=0,00055 \mathrm{~T}^{2,63}\end{array}$ & $\begin{array}{c}\text { Brody Davis } \\
\text { et Ragsdale (I937) } \\
\text { Hvidsten (I } 9.40 \text { ) }\end{array}$ \\
\hline
\end{tabular}

De plus, à la suite des travaux initiaux de Brody et collaborateurs (5 et 6), SteEnsberg et Ostergaard (4) ont fait intervenir dans leur formule : 1'âge en mois ; l'expression qu'ils recommandent devient alors : $\mathrm{P}=a \mathrm{~T}^{n} \mathrm{~A}^{m}, \mathrm{~A}$ étant l'âge en mois, $m$, un exposant constant. STEENSBERG et OsTERGAARD ont ainsi établi des formules précises pour des animaux des races Rouge Danoise, Pie Noire Danoise et Shorthorn, soumises à 3 niveaux alimentaires différents. Nous avons vu précédemment comment ces auteurs avaient matérialisé ces résultats théoriques pour une utilisation pratique.

MARCQ et LAHAYe (7), puis LeRoy (8) ont signalé à l'intention des éleveurs la formule simple de CREVAT : $\mathrm{P}=\mathrm{K} \mathrm{T}^{3}$; $\mathrm{T}$ est le tour de poitrine 
en mètres ; $K$ est un coefficient qui a été chiffré en moyenne à 8o mais qui dépend de l'âge et de l'état des animaux; il devient :

- Ioo pour les veaux;

- 90 pour les jeunes d'élevage ;

- 85 pour les bœufs maigres;

- 80 pour les bœufs en état;

- 76 pour les bœufs mi-gras ;

- 72 pour les boufs gras;

- 68 pour les bœufs fin gras.

$4^{\circ}$ L'estimation pratique $\mathrm{du}$ poids vif en fonction $\mathrm{du}$ tour de poitrine.

Dans la pratique, on a le choix entre l'utilisation d'une équation curvilinéaire et celle d'une série de droites de régression établies par classes d'âge ou classes de tour de poitrine ; Johansson et HiLDEMaN préconisent la première méthode pour des animaux en croissance - car il est bien connu que la croissance des jeunes animaux est curvilinéaire - la deuxième convient de préférence pour les animaux adultes.

Dans les deux cas, les écarts-types des déviations des points expérimentaux à la courbe d'une part, et aux droites de régression d'autre part sont comparables. JoHansson et HiLdeman (I) en essayant de chiffrer l'importance de l'erreur obtenue par détermination du poids vif à partir du tour de poitrine, estiment qu'elle est, en moyenne, du 6 p. Ioo du poids vif (ce qui correspond, par exemple, à une erreur de $30 \mathrm{~kg}$ pour une vache pesant environ $500 \mathrm{~kg}$ ). Cette erreur pourrait être réduite si l'on travaillait sur une population homogène ou sur des groupes d'animaux comparables, tant par leur condition que par leur âge.

\section{$5^{\circ}$ Les sources d'erreur dans l'utilisation des formules de barymétrie.}

Les sources d'erreur provenant de l'utilisation des formules de barymétrie sont, d'une part, des erreurs physiques de pesée et de mensuration, d'autre part, des erreurs statistiques dues à l'ajustement de données individuelles à une équation de régression, chaque animal ayant une conformation qui lui est propre.

I,USH et COPEr,aND (9) ont étudié les erreurs faites sur diverses mensurations corporelles répétées I f fois sur chaque animal, de 2 groupes de 9 vaches et Io génisses de race Jerseyaise. Pour le tour de poitrine, cette erreur, exprimée en pourcentage de la mesure moyenne était de 0,83 p. Ioo pour les vaches et de $0,93 \mathrm{p}$. Ioo pour les génisses.

Les variations du poids vif dans une courte période, et les erreurs de pesée qu'elles entraînent ont fait l'objet de nombreuses études à la suite des travaux de base de Christensen, Wilson et Brack (Io), MAyMone et SiRcana (II).

En fait, d'après Johansson et Hildeman (I) l'erreur de pesée est 
approximativement de I p. roo lorsque celle-ci est effectuée à des heures régulières et de $2 \mathrm{p}$. Ioo dans les autres cas.

En conclusion, on peut dégager de l'ensemble des données recueillies jusqu'à ce jour que l'erreur, dans la détermination du poids vif est due, pour moitié à des erreurs physiques, et pour moitié aux erreurs statistiques que nous avons explicitées précédemment.

\section{$6^{\circ}$ Les autres relations :}

Divers auteurs ont établi des relations pratiques de barymétrie entre le poids vif et d'autres mensurations corporelles. Dans la plupart d'entre elles, le corps de l'animal est assimilé à un cylindre de révolution. Nous nous contenterons de rappeler l'inventaire de ces formules de barymétrie dressé par Marco et LAHAYe (7).

- La formule de CREVAT:

$$
\mathrm{P}=80 \cdot \mathrm{T} \cdot \mathbf{L} \cdot \mathrm{V} .
$$

( $\mathrm{I}$, est la longueur du corps,

$\mathrm{T}$, le tour de poitrine,

$\mathrm{V}$, le tour ventral, exprimés tous trois en mètres).

- La formule de Qú́tELET:

$$
\mathrm{P}=87,5 \cdot \mathrm{T}^{2} \cdot \mathrm{L} .
$$

(' $T$ et $L$ ont la même signification que précédemment).

- La formule de MatiÉwitch :

$$
\mathrm{P}=\left(\frac{\mathrm{T}+\mathrm{V}}{2}\right)^{2} \cdot \mathrm{M} \cdot 62 .
$$

( $\mathrm{T}$ et $\mathrm{V}$ gardent la même signification; $\mathrm{M}$ est la longueur sternoilio- ischiale en $\mathrm{m}$ ).

- Autre formule de Crevat :

En outre, CREVAT a donné une formule de barymétrie dans laquelle intervient le tour spiral:

$$
\mathrm{P}=40 \mathrm{~F}^{3} .
$$

(F est le tour spiral en $\mathrm{m}$ ).

Cette mesure, très rarement mentionnée par les auteurs, paraît cependant avoir un réel intérêt, car elle donne une bonne idée du volume de l'animal et, en particulier, du développement de ses parties postérieures. L'allure de celles-ci est, en effet, modifiée par l'état d'engraissement qui introduit, nous l'avons vu, de notables erreurs dans la détermination du poids vif en fonction du tour de poitrine. Sa mesure, pour avoir une bonne précision, doit être effectuée des deux côtés de l'animal. Rappelons qu'elle nécessite deux opérateurs et que le ruban, fixé à la pointe du sternum par le premier opérateur, doit passer au milieu du 
bras (droit ou gauche), à la limite dos-rein, à un travers de main sous la pointe de la hanche (du côté opposé à celui du bras) et se terminer horizontalement un travers de main en dessous du périnée.

\section{III. - Nos RÉSULTATS}

Nous avons travaillé sur un ensemble de $20 \mathrm{r}$ données provenant d'animaux des races Française Frisonne Pie Noire, Normande et Pie Rouge de 1'Est, présentés aux Concours généraux de 1952 et I954.

\section{Corrélations entre le poids vif et certaines mensurations corporelles :}

Une première étude des corrélations entre le poids vif et quatre mensurations (tour de poitrine, tour spiral, hauteur de poitrine et longueur du corps) chez les mâles de ces différentes races, nous a montré l'intérêt primordial du tour de poitrine et du tour spiral par rapport aux deux autres mensurations. Tous ces résultats apparaissent dans le tableau $V$, où est indiquée également la valeur du coefficient de corrélation entre, le poids vif et la moyenne arithmétique du tour de poitrine et du tour spiral.

\section{Tableau V}

Coefficients de corrélation entre le poids vif et certaines mensurations pour les taureaux.

\begin{tabular}{|c|c|c|c|c|c|c|}
\hline Race & $\begin{array}{c}\text { Nombre } \\
\text { d'animaux }\end{array}$ & de $\begin{array}{ll}\text { Tour } \\
\text { poitrine }\end{array}$ & $\begin{array}{l}\text { Tour } \\
\text { spiral }\end{array}$ & $\begin{array}{c}\text { Moyenne } \\
\text { tour } \\
\text { de poitrine } \\
\text { tour spiral }\end{array}$ & $\begin{array}{l}\text { Longueur } \\
\text { du corps }\end{array}$ & $\begin{array}{c}\text { Hauteur } \\
\text { au garrot }\end{array}$ \\
\hline $\begin{array}{l}\text { Normande } \ldots \ldots \ldots \ldots \ldots \\
\text { F. F. P. N. }{ }^{2} \ldots \ldots \ldots \ldots \\
\text { Pie rouge de l'Est } \ldots \ldots \ldots\end{array}$ & $\begin{array}{l}26 \\
19 \\
29\end{array}$ & $\begin{array}{l}0,98 \\
0,98 \\
0,98\end{array}$ & $\begin{array}{l}0,97 \\
0,97 \\
0,98\end{array}$ & $\begin{array}{l}0,98 \\
0,98 \\
0,99\end{array}$ & $\begin{array}{l}0,81 \\
0,86 \\
0,47\end{array}$ & $\begin{array}{l}0,79 \\
0,86 \\
0,64\end{array}$ \\
\hline
\end{tabular}

$2^{\circ}$ Coefficients de régression linéaire du poids vif sur le tour de poitrine et le tour spiral :

Les coefficients de régression du poids vif sur le tour de poitrine, le tour spiral et la moyenne arithmétique de ces deux mensurations ont ensuite été calculés. Les résultats obtenus sont rassemblés dans le tableau VI.

Ces valeurs de coefficients de régression provenaient de lots d'animaux dont les poids moyens étaient différents et où les écarts de poids individuels étaient importants $(450$ à $\mathrm{I} 250 \mathrm{~kg}$ dans la plupart des lots de taureaux). Une étude complète de cette question aurait exigé une analyse précise des différents facteurs agissant sur ce coefficient de 


\section{TABLEAU VI}

Coefficients de régression du poids vif sur le tour de poitrine, le tour spiral et leur moyenne arithmétique

\begin{tabular}{|c|c|c|c|c|c|c|}
\hline Race & Sexe & $\begin{array}{l}\text { Nombre } \\
\text { danimaux }\end{array}$ & $\begin{array}{l}\text { Poids } \\
\text { moyen }\end{array}$ & $\begin{array}{l}\text { Tour de } \\
\text { poitrine }\end{array}$ & $\begin{array}{l}\text { Tour } \\
\text { spiral }\end{array}$ & $\begin{array}{c}\text { Moyenne } \\
\text { arithmétique }\end{array}$ \\
\hline \multirow{2}{*}{ Normande..... } & Mâle & 26 & 889,9 & 9,02 & 8,40 & 8,76 \\
\hline & $\begin{array}{l}\text { Femelle } \\
\text { Yâle }\end{array}$ & 48 & 719,7 & 7,56 & 7,33 & 7,40 \\
\hline F. F. P. N. . . & Femelle & 19 & $\begin{array}{l}8 \div 3,8 \\
612,2\end{array}$ & $\begin{array}{l}8,78 \\
8,04\end{array}$ & $\begin{array}{l}7,98 \\
6,99\end{array}$ & $\begin{array}{l}8,72 \\
7,11\end{array}$ \\
\hline \multirow[t]{2}{*}{ Pie rouge..... } & IIale & 29 & 800,4 & 9,03 & 8,28 & 8,77 \\
\hline & Femelle & $4+$ & 662,4 & 8,025 & $5 \cdot 39$ & 6,82 \\
\hline
\end{tabular}

régression (race, sexe, âge, condition), analyse que le nombre limité de nos résultats ne nous permettait pas d'envisager.

Ces résultats confirment cependant que les coefficients de régression sont plus élevés dans le sexe mâle que dans le sexe femelle.

A titre d'exemple nous avons montré par ailleurs à partir de la régression du poids vif sur le tour de poitrine l'intérêt qu'il y aurait à séparer les animaux mâles en deux classes de tour de poitrine correspondant à des classes de poids s'étalant respectivement de 450 à 800 $850 \mathrm{~kg}$, et de $800-850$ à I $250 \mathrm{~kg}$. La somme des carrés des écarts entre les données expérimentales et les valeurs d'ajustement aux deux portions de droite de régression est nettement inférieure à celle obtenue à partir d'une seule droite de régression, comme le prouvent les résultats du tableau VII.

\section{TABLEAU VII}

\begin{tabular}{|c|c|c|c|c|c|}
\hline \multirow{2}{*}{ Race } & \multicolumn{3}{|c|}{$\begin{array}{l}\text { Coefficients de régression } \\
\text { du poids vif sur le tour de poitrine }\end{array}$} & \multicolumn{2}{|c|}{$\begin{array}{l}\text { Somme des carrés des écarts } \\
\text { des valeurs d'ajustement } \\
\text { aux valeurs expérimentales }\end{array}$} \\
\hline & $1^{\text {te }}$ classe & $2^{\mathrm{e}}$ classe & Ensemble & $\begin{array}{l}\text { Cas d'i droite } \\
\text { de régression }\end{array}$ & $\begin{array}{c}\text { Cas de } 2 \text { droites } \\
\text { de régression }\end{array}$ \\
\hline $\begin{array}{l}\text { Normande } \ldots \ldots \ldots \ldots \ldots \\
\text { F. F. P. N. } \ldots \ldots \ldots \ldots \ldots \\
\text { Pie rouge } \ldots \ldots \ldots \ldots\end{array}$ & $\begin{array}{l}7,89 \\
7,49 \\
8,08\end{array}$ & $\begin{array}{r}10,32 \\
9,29 \\
10,82\end{array}$ & $\begin{array}{l}9,02 \\
8,-8 \\
9,03\end{array}$ & $\begin{array}{l}20,9 \text { 10 } \\
28,150 \\
38,800\end{array}$ & $\begin{array}{l}14,100 \\
24,040 \\
31,030\end{array}$ \\
\hline
\end{tabular}

\section{$3^{\circ}$ Valeur statistique des trois équations de régression linéaire.}

Nous avons enfin essayé de comparer la précision statistique obtenue en utilisant chacune des trois droites de régression du poids vif sur le tour de poitrine, le tour spiral et leur moyenne arithmétique. Nous l'avons chiffrée par l'écart-type des déviations des valeurs individuelles observées aux valeurs théoriques d'ajustement. Les résultats sont consignés dans le tableau VIII. 


\section{TABLEAU VIII}

Ecarts-types des déviations des valeurs expérimentales par rapport aux valeurs théoriques d'ajustement (exprimés en valeurs absolues et en pourcentages des poids vifs moyens).

\begin{tabular}{|c|c|c|c|c|c|c|c|}
\hline \multirow[b]{2}{*}{ Race } & \multirow[b]{2}{*}{ Sexe } & \multicolumn{2}{|c|}{ Tour de poitrine } & \multicolumn{2}{|c|}{ Tour spiral } & \multicolumn{2}{|c|}{ Moyenne arithmétique } \\
\hline & & $\begin{array}{l}\text { Ecarts- } \\
\text { types en } \\
\text { valeurs } \\
\text { absolues }\end{array}$ & $\begin{array}{c}\text { Ecarts- } \\
\text { types en } \% \\
\text { des poids } \\
\text { vifs moyens }\end{array}$ & $\begin{array}{l}\text { Écarts- } \\
\text { types en } \\
\text { valeurs } \\
\text { absolues }\end{array}$ & $\begin{array}{c}\text { Ecarts- } \\
\text { types en } \% \\
\text { des poids } \\
\text { vifs moyens }\end{array}$ & $\begin{array}{l}\text { Écarts- } \\
\text { types en } \\
\text { valeurs } \\
\text { absolues }\end{array}$ & $\begin{array}{l}\text { Ecarts- } \\
\text { types en \% } \\
\text { des poids } \\
\text { vifs moyens }\end{array}$ \\
\hline $\begin{array}{l}\text { Normande.... } \\
\text { F. F. P. ..... } \\
\text { Pie rouge.... }\end{array}$ & $\begin{array}{c}\text { Mâle } \\
\text { Femelle } \\
\text { Mâle } \\
\text { Femelle } \\
\text { Mâle } \\
\text { Femelle }\end{array}$ & $\begin{array}{l}59,6 \\
48,8 \\
43,6 \\
34,6 \\
31,15 \\
50,3\end{array}$ & $\begin{array}{l}6,7 \\
6,8 \\
5,0 \\
5,6,5 \\
3,9 \\
7,6\end{array}$ & $\begin{array}{l}39,0 \\
52,8 \\
50,3 \\
42,85 \\
55,85 \\
49,15\end{array}$ & $\begin{array}{l}4,4 \\
7,35 \\
5,75 \\
7,0 \\
7,0 \\
7,4\end{array}$ & $\begin{array}{l}49,95 \\
50,1 \\
38,5 \\
34,15 \\
36,3 \\
57,3\end{array}$ & $\begin{array}{l}5,6 \\
6,95 \\
4,4 \\
5,6 \\
4,55 \\
8,65\end{array}$ \\
\hline
\end{tabular}

Ces écarts-types représentent, en pourcentage du poids vif moyen, une erreur relative de 3,9 à $8,6 \mathrm{p}$. Ioo ; en ce qui concerne les écartstypes par rapport à la droite de régression calculée à partir du tour de poitrine, les erreurs relatives extrêmes de 3,9 à $7,6 \mathrm{p}$. Ioo sont très voisines de celles qui sont indiquées par la plupart des auteurs.

I,e tour de poitrine donne, dans l'ensemble, la meilleure précision statistique d'ajustement et l'utilisation de la moyenne arithmétique tour de poitrine-tour spiral, ne permet pas de réduire sensiblement l'amplitude des déviations des valeurs expérimentales aux valeurs théoriques.

Le tableau IX rassemble les diverses équations de régression du poids vif sur le tour de poitrine que nous avons obtenues pour les animaux

\section{TABLEAU IX}

Droites de régression du poids vif sur le tour de poitrine $\mathrm{Y}=$ poids vif en $\mathrm{kg}$.

$\mathrm{X}=$ tour de poitrine en $\mathrm{cm}$.

\begin{tabular}{|c|c|c|}
\hline Race & Sexe & Équation \\
\hline $\begin{array}{l}\text { Normande } \ldots \ldots \ldots \ldots \ldots \\
\text { F. F. P. N. } \ldots \ldots \ldots \ldots \ldots \ldots \\
\text { Pie rouge } \ldots \ldots \ldots \ldots \ldots \ldots \ldots\end{array}$ & $\begin{array}{l}\text { Mâle } \\
\text { Femelle } \\
\text { Mâle } \\
\text { Femelle } \\
\text { Mâle } \\
\text { Femelle }\end{array}$ & 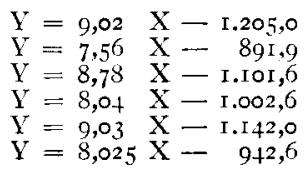 \\
\hline
\end{tabular}

mâles et femelles des trois races étudiées. Ces équations sont susceptibles d'une utilisation pratique pour des sujets dont le poids vif est supérieur à $45^{\circ} \mathrm{kg}$, et qui seraient dans un état d'embonpoint comparable à celui d'animaux de concours. 
$4^{\circ}$ Equation curvilinéaire de régression du poids vif sur le tour de poitrine.

Après cette étude préliminaire, nous nous sommes attachés à rechercher la formule qui donnerait la meilleure estimation du poids vif en fonction du tour de poitrine. Les recherches antérieures ayant montré l'intérêt des relations de la forme : $\mathrm{P}=a \mathrm{~T}^{n}, \mathrm{P}$ étant le poids vif en $\mathrm{kg}$, $\mathrm{T}$, le tour de poitrine en $\mathrm{cm}$, nous avons calculé l'équation de régression des logarithmes du poids vif sur les logarithmes du tour de poitrine. On a, en effet $: \log \mathrm{P}=n \log \mathrm{T}+\log a$. Le tableau $\mathrm{X}$ donne les valeurs des constantes $a$ et $n$, obtenues sur l'ensemble des animaux des races Normande, Française Frisonne Pie Noire et Pie Rouge de 1'Est.

Tableau $X$

\begin{tabular}{|c|c|c|}
\hline Race & $n$ & $\mathrm{a}$ \\
\hline $\begin{array}{l}\text { Normande } \ldots \ldots \ldots \ldots \ldots \\
\text { F.F.P.N. } \ldots \ldots \ldots \ldots \ldots \\
\text { Pie rouge de l'est } \ldots \ldots\end{array}$ & $\begin{array}{l}2,7 \mathrm{I} \\
2,68 \\
2,60\end{array}$ & $\begin{array}{l}0,00034 \\
0,0003^{6} \\
0,00049\end{array}$ \\
\hline
\end{tabular}

Ces données sont tout à fait comparables à celles qui sont citées dans les études des autres auteurs.

$5^{\circ}$ Comparaison des régressions linéaire et curvilinéaire du poids vif sur le tour de poitrine.

Il nous restait enfin à comparer ces différents modes d'estimation du poids vif des bovins en fonction de leur tour de poitrine : droite de régression et équation curvilinéaire. Les degrés de précision obtenus dans les deux cas ont été exprimés par l'écart-type des déviations des valeurs expérimentales par rapport aux valeurs théoriques d'ajustement. Le tableau XI permet de comparer ces résultats pour les animaux de race Normande.

\section{TABLEAU XI}

Comparaison des ajustements

aux courbe et droite de régression du poids vif

sur le tour de poitrine pour les animaux de race Normande.

\begin{tabular}{|c|c|c|c|c|}
\hline & \multicolumn{2}{|c|}{ Taureaux } & \multicolumn{2}{|c|}{ Vaches } \\
\hline & écart-type & $\%$ du poids moyen & écart-type & $\%$ du poids moyen \\
\hline $\begin{array}{l}\text { Droite de régression } \\
\text { Courbe............ }\end{array}$ & $\begin{array}{l}55 \mathrm{~kg} \\
49 \mathrm{~kg}\end{array}$ & $\begin{array}{l}6,2 \% \\
5,5 \%\end{array}$ & $\begin{array}{l}38 \mathrm{kgr} \\
43 \mathrm{~kg}\end{array}$ & $\begin{array}{l}5,7 \% \\
6,4 \%\end{array}$ \\
\hline
\end{tabular}


On peut en conclure que si l'ajustement à une droite est préférable pour les vaches, vraisemblablement par suite de l'amplitude restreinte de la variation de leurs poids vifs, l'équation curvilinéaire demeure, par contre, à utiliser pour les taureaux, pour la raison inverse.

\section{IV. - CONCLUSIONS}

Une première étude comparative des diverses droites de régression du poids vif sur le tour de poitrine, le tour spiral et la moyenne arithmétique tour de poitrine-tour spiral, nous a montré l'intérêt de la méthode basée sur l'emploi du tour de poitrine, qui fournit l'ajustement linéaire le plus commode et le plus précis.

Cependant, la valeur de cette méthode d'évaluation à partir de la droite de régression du poids vif sur le tour de poitrine est liée à l'amplitude des variations de poids de chaque lot; une répartition très étalée des données, ou incluant de jeunes sujets, rend préférable 1'utilisation d'une équation curvilinéaire ou d'une série de droites de régression correspondant à plusieurs classes de tour de poitrine.

L'erreur relative faite, lors de l'estimation du poids vif, par l'intermédiaire du tour de poitrine, est de l'ordre de 5 à 6 p. Ioo. Cependant, avec des mensurations et des pesées plus précises et des lots de sujets plus homogènes, il serait vraisemblablement possible de réduire cette approximation qui, dans le cas de l'appréciation du poids d'animaux de boucherie destinés à être vendus, se trouve évidemment trop importante.

Pour mieux expliciter l'ensemble de nos résultats statistiques, il serait intéressant de suivre, en détail, la croissance de jeunes bovins jusqu'à un âge adulte et de voir ce qui, dans la différence de conformation de deux animaux ayant même tour de poitrine, retentit sur leur poids vif. Des équations de régression multiple où figureraient les deux ou trois mensurations les plus étroitement liées au poids vif, permettraient alors de diminuer sensiblement les erreurs statistiques que nous avons mentionnées.

\section{RÉFÉRENCES BIBLIOGRAPHIQUES}

(I) Johansson (I.), Hildeman (S. E.). - The relationship between certain body measurements and live and slaughter weight in cattle. Animal Breeding Abstracts, I954, vol. 22, no I, p. I-I7.

(2) Branton (C.), Salisbury (G. W.). The estimation of the weight of bulls from girth measurements. Journal of Dairy Science, I946, vol. 29, $\mathrm{n}^{\circ}$ 3, p. I $4 \mathrm{I}-\mathrm{I} 43$.

(3) OstergaARd (P. S.). - Undersøgelser vedrørende jerseykvaegets vaegt, al og ydelse. Beretning fra forsogslaboratoriet, I950, $\mathrm{n}^{0}$ 25I, Kobenhavn.

(4) Steensberg (V.), OstergaArd (P. S.). - Foderenhedsmaengdens indflydelse paa ungkvaegets vaekst II. Beretning fra forsogslaboratoriet, I945, no 2 I6, Kobenhavn. 
(5) Brody (S.), Davis (H. P.), Ragsdale (A. C.). - Growth and development with special reference to domestic animals. Missouri Station Research Bulletin $1937, \mathrm{n}^{\circ} 262$, p. $24, \mathrm{n}^{\circ} 263$, p. 40.

(6) BRODY (S.). - Bioenergetics and growth. Reinhold publishing corporation, I945, New-York.

(7) Marce (J.), Lahaye (J.). - Les Bovins. Tome I : Connaissance du bétail. Librairie agricole de la Maison Rustique, I935, Paris.

(8) LERoy (A. M.). - Le Bœuf. Encyclopédie des connaissances agricoles. Librairie Hachette, I942, Paris.

(9) Lush (J. L.), Copeland (O. C.). - A study of the accuracy of measurements of dairy cattle. Journal of Agricultural Research, 1930, n 4 r, p. $37-49$.

(io) Lush (J. L.), Christensex (F. W.), Wilsox (C. V.), Black (W. H.). The accuracy of cattle weight. Journal of Agricultural Research, I948, $\mathrm{n}^{0} 36$, p. 55I-579.

(II) Maymone (B.), SircaNa (C.). - Le variazioni normali del peso vivo nei bovini. Annali dell' Instituto Sperimentale Zootecnico di Roma, I929, vol. I. 